\title{
Tumor-Cell-Surface Adherable Peptide-Drug Conjugate Prodrug Nanoparticles Inhibit Tumor Metastasis and Augment Treatment Efficacy
}

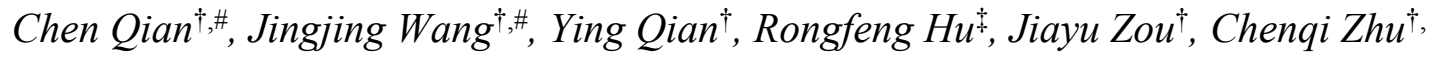

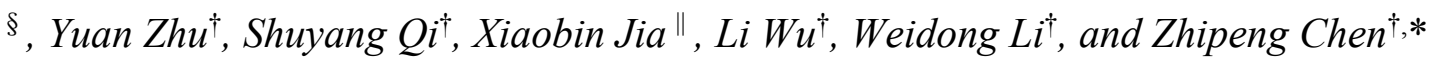

†College of pharmacy, Nanjing University of Chinese Medicine, Nanjing 210023,

China

ॠKey Laboratory of Xin’an Medicine, Ministry of Education, Anhui Province Key

Laboratory of R\&D of Chinese Medicine, Anhui University of Chinese Medicine,

Hefei, Anhui 230038, China.

$\S$ Department of Pharmacy, the Affiliated Suzhou Hospital of Nanjing Medical

University, Suzhou 215002, China

" School of Traditional Chinese Pharmacy, China Pharmaceutical University, Nanjing 211198, China

${ }^{\#}$ Chen Qian, Jingjing Wang and Ying Qian contributed equally to this work.

*Address correspondence to:

czpcpu2000@hotmail.com. Tel: 025-85811050. 
Figure S1. Binding of Pep in the active site of MMP-2 protein (1HOV) cavity as assessed by molecular docking software technology SYBYL.

Figure S2. The synthesis of (A)DOX-SH, (B)Pep-S-S-Py and (C)PDC.

Figure S3. The HPLC and MS of (A) CKIGLFRWR, (B) CCKIGLFRWR, (C) KIGLCCFRWR, (D) KIGLFRWRCC, (E) DOX-SH, (F) Pep-S-S-Py, (G) PDC.

Figure S4. Fluorescence excitation spectra of pyrene in PDC aqueous solution

Figure S5. The structural formula and 1H NMR spectrum of the MPGA. The graft rate of maleic anhydride was $77.6 \%$.

Figure S6. The CD spectrum of the PDC.

Figure S7. The extracellular aggregation of PDC-FITC within $12 \mathrm{~h}$.

Figure S8. In vitro release profiles of DOX-SH from degradable PDC in different reducing and acidic environments

Figure S9. Quantitative analysis by flow cytometry of the intracellular DOX-SH that 
was released by cell surface cleavage.

Figure S10. In vitro antitumor effects of Pep, DOX, DOX-SH and PDC at different concentrations in SMMC-7721 cells.

Figure S11. The quantitative analysis of invasion rate through wound healing assays within $24 \mathrm{~h}$ of SMMC-7721 cells.

Figure S12. The inhibition of longitudinal motility ability of SMCC-7721 cells influenced by positive drug Batimastat.

Figure S13. The quantitative analysis of the inhibition rate through transwell assays of SMMC-7721 cells.

Figure S14. Quantitative analysis of the expression of E-cadherin and Caspase-3.

Figure S15. Quantitative analysis of the expression of MMP1, MMP2, Pro-MMP2, MMP9 and Pro-MMP9.

Figure S16. Pharmacokinetic behavior of free DOX, PDC and CSN NPs in mice after intravenous administration of different DOX formulations at the DOX dose of $4 \mathrm{mg} / \mathrm{kg}$ over a span of $48 \mathrm{~h}$. The data are presented as the mean $\pm \operatorname{SD}(n=6)$.

Figure S17. In vivo fluorescence imaging (A) and fluorescence intensity (B) of the DiR signal from HCCLM3-LUC tumor-bearing BALB/c mice after intravenous injection 
with the Cy7-labeled PDC and the Cy7-labeled CSN NPs at different time points. The dose of DOX was $6 \mathrm{mg} / \mathrm{kg}$ based on the animal's weight.

Figure S18. Ex vivo fluorescence images of important tissues collected from the mice $72 \mathrm{~h}$ after administration.

Figure S19. Fluorescence imaging of tumor tissue after $72 \mathrm{~h}$ post injection.

Figure S20. H\&E analyses for main organs collected on day 27 after first injection

Table S1. Pharmacokinetic parameters of DOX, PDC, and CSNs in rats plasma

\section{Figure S1}

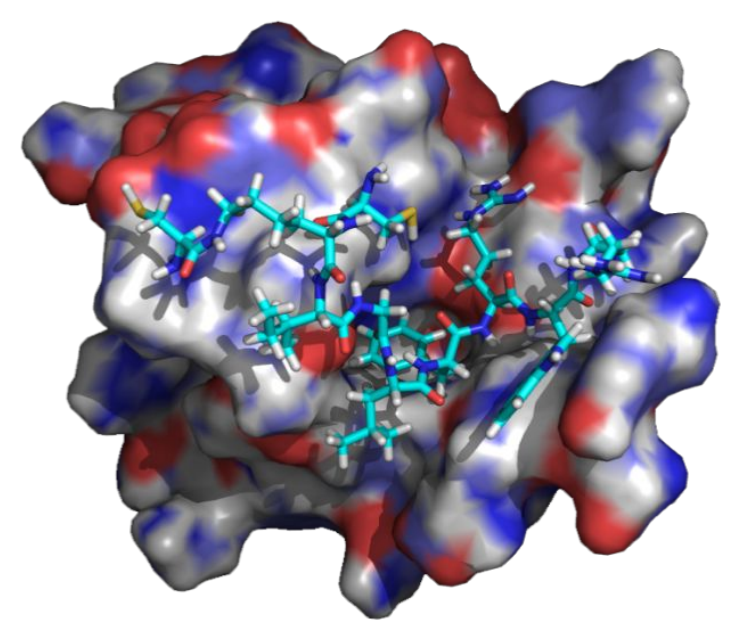

\section{Figure S2}

(A) 

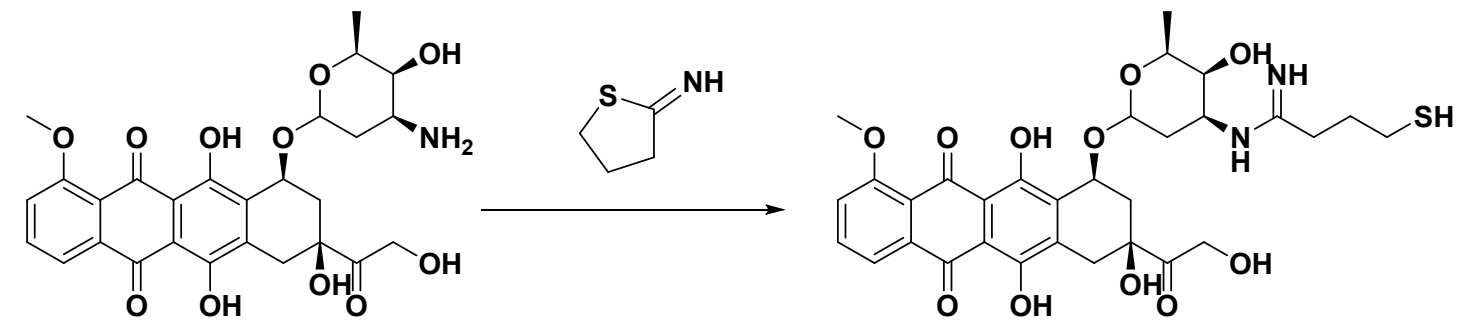

(B)

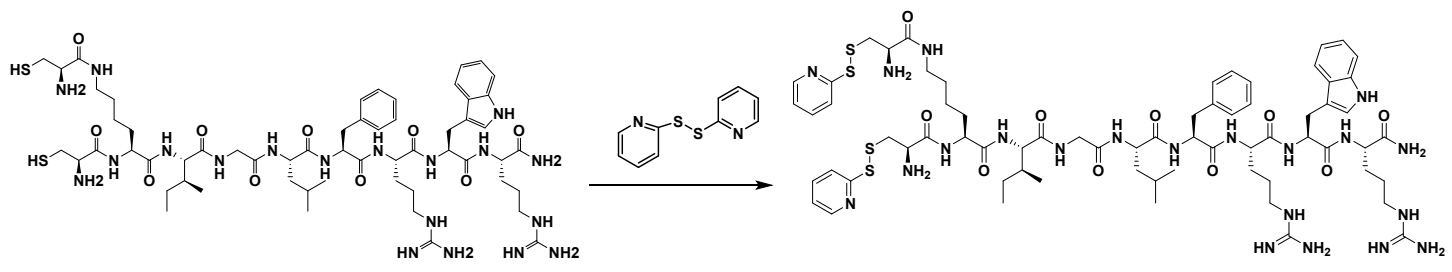

(C)

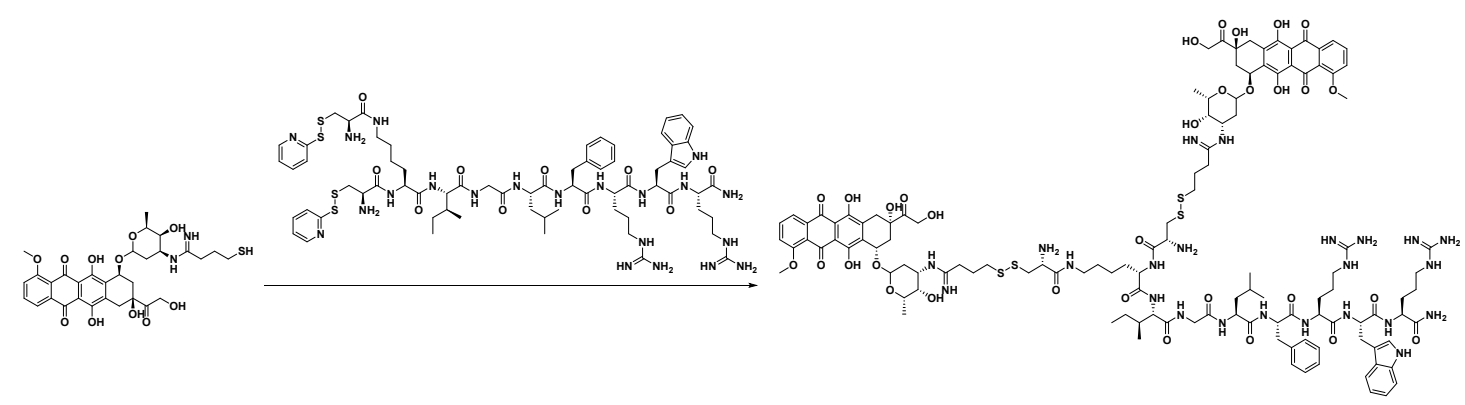

Figure S3

(A) CKIGLFRWR
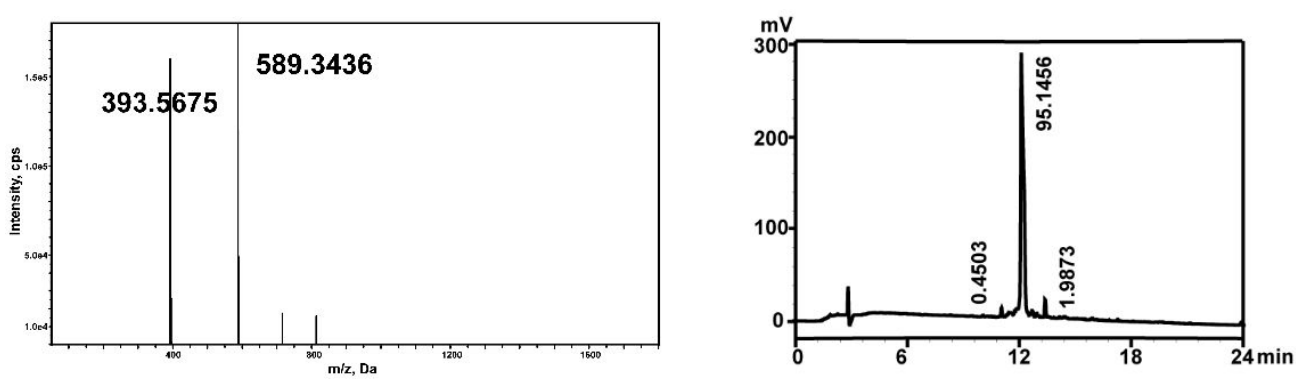

(B) CCKIGLFRWR 

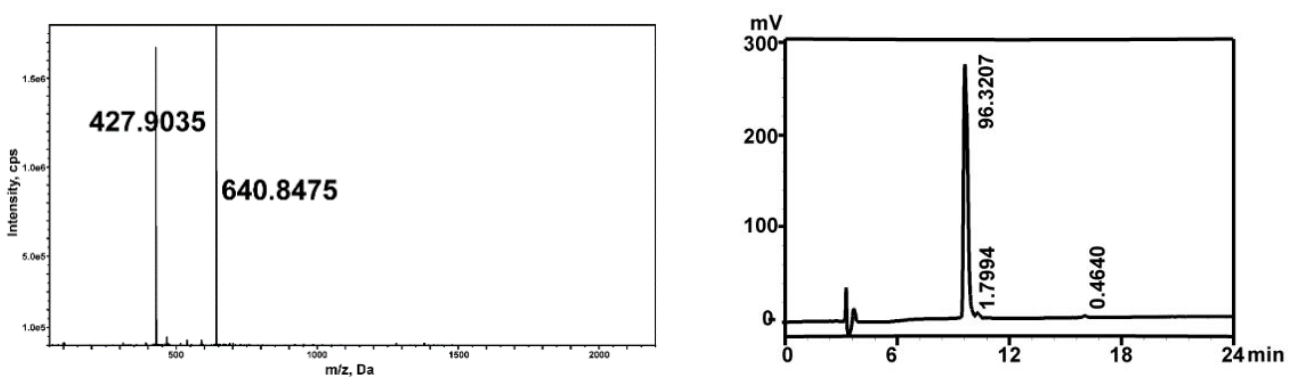

(C) KIGLCCFRWR
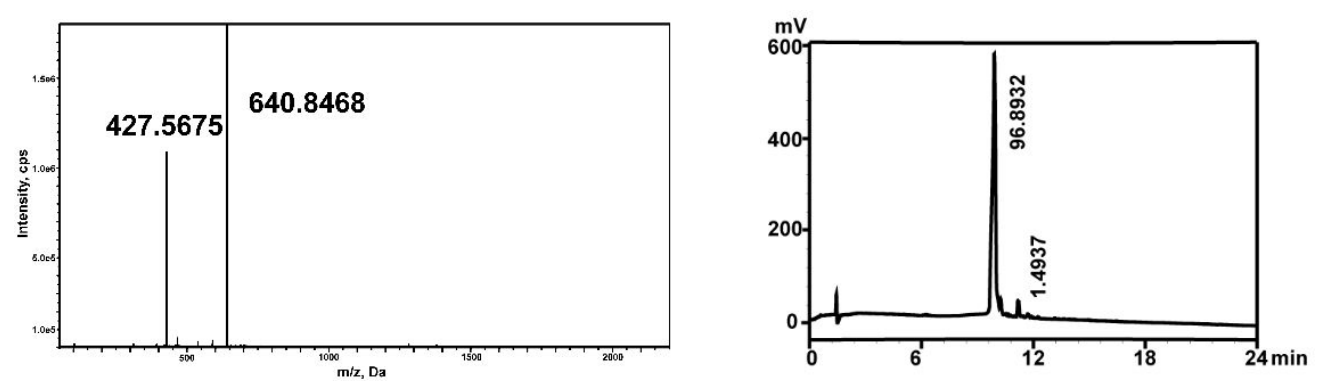

\section{(D) KIGLFRWRCC}
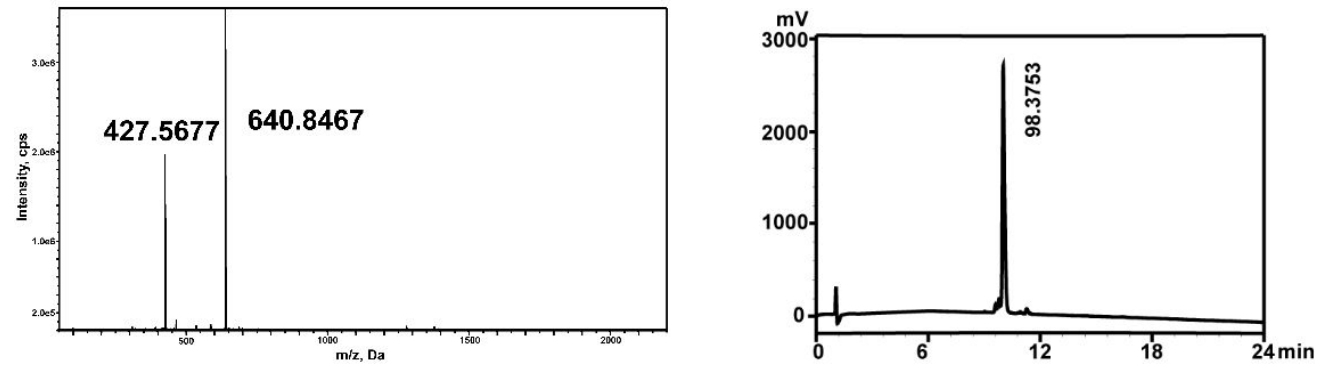

(E) DOX-SH
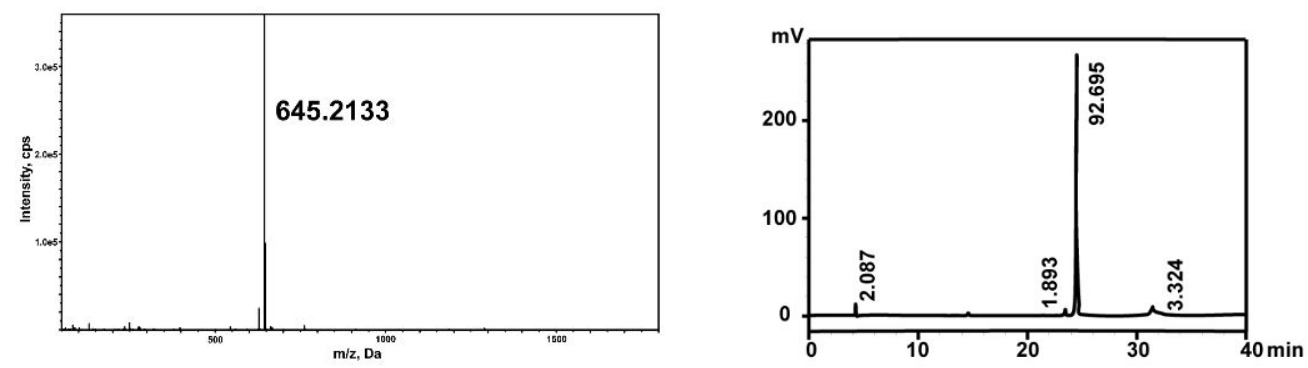

(F) Pep-S-S-Py 

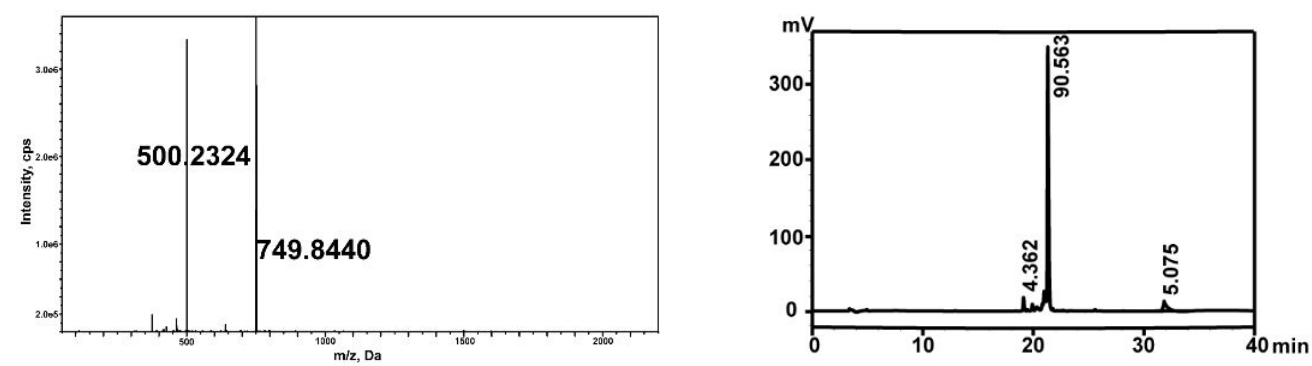

(G) PDC
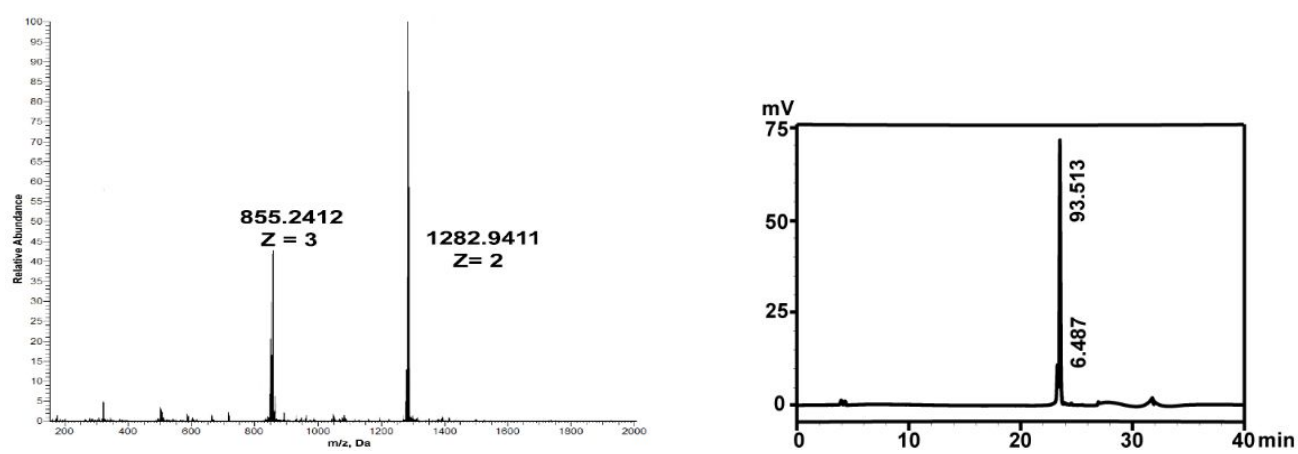

Figure S4

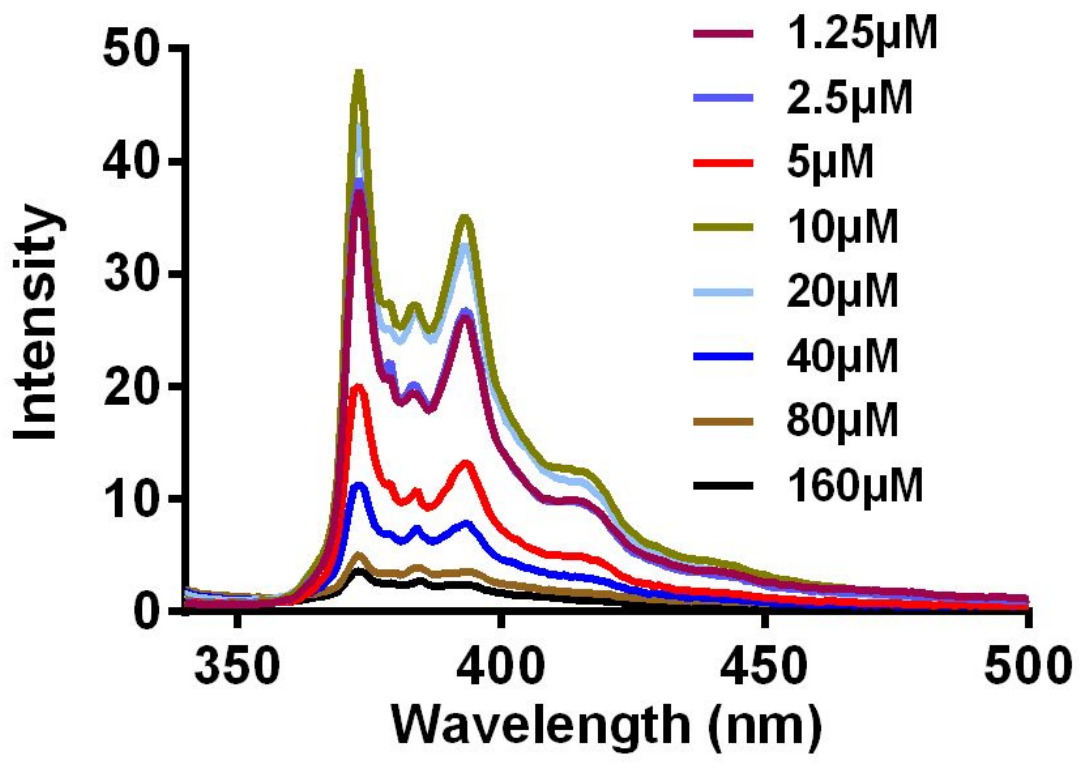

Figure S5 


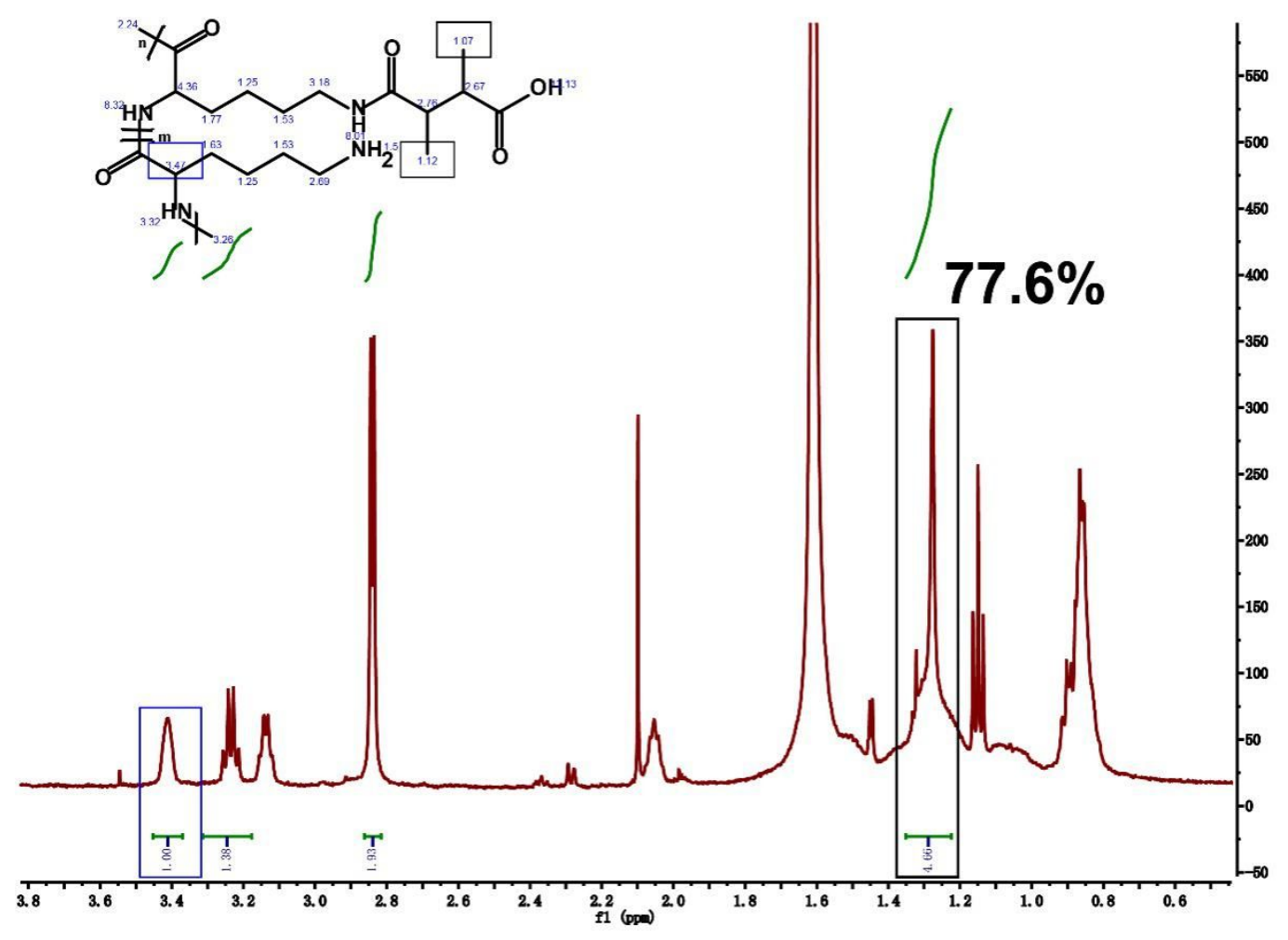

Figure S6

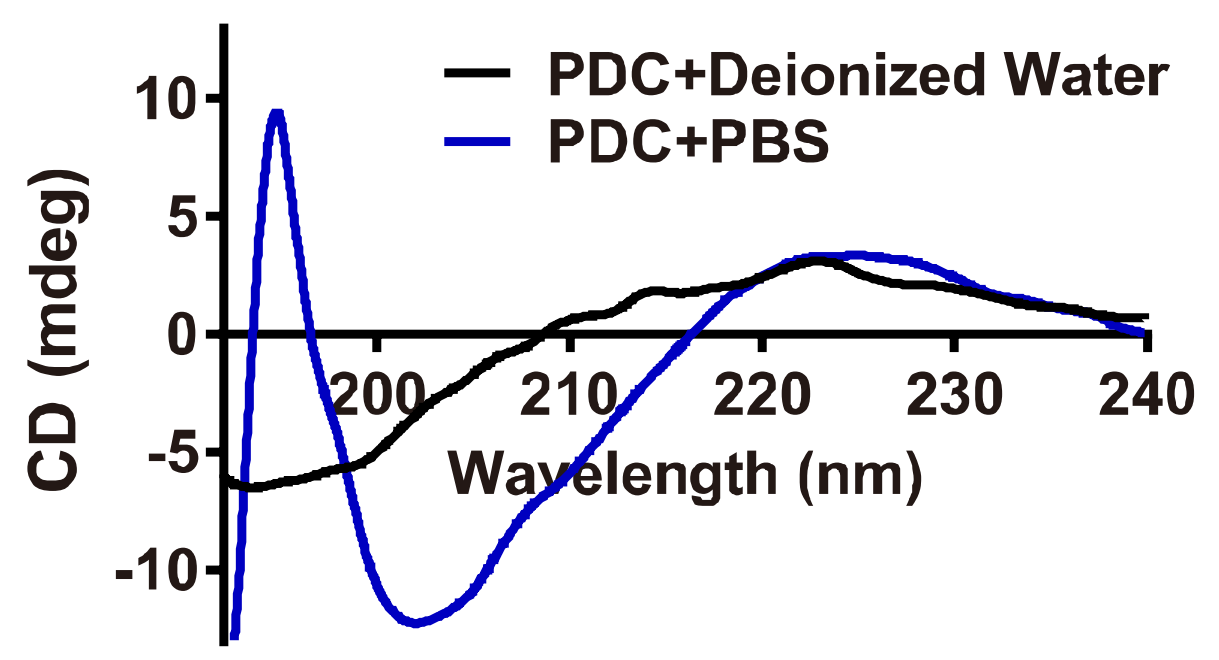

Figure S7 


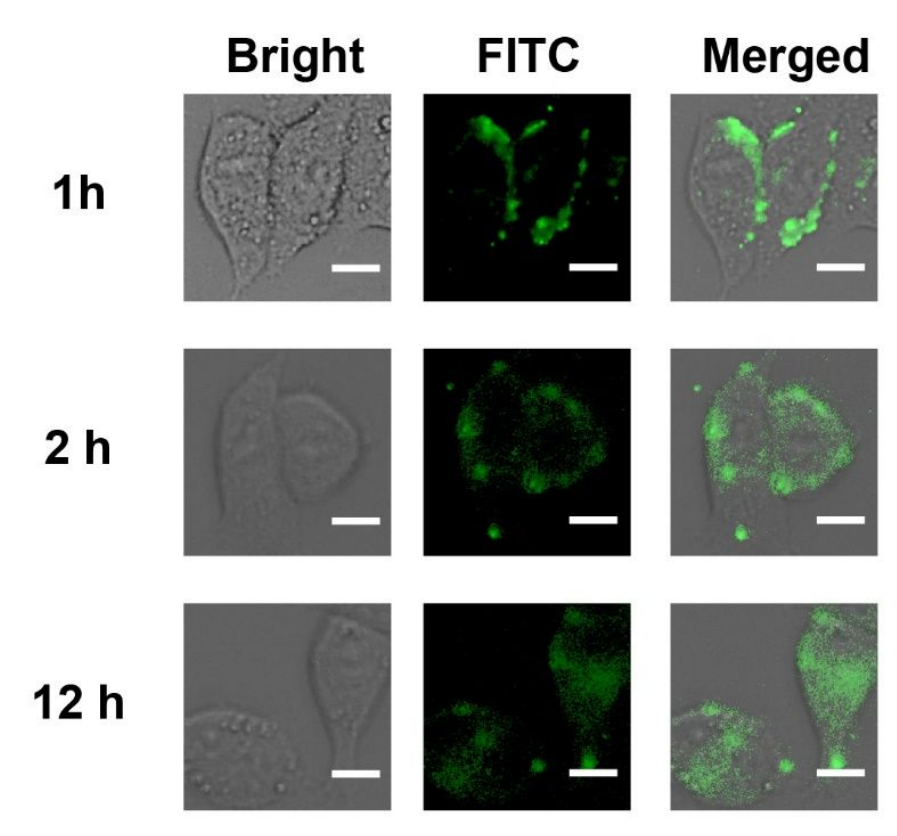

Figure S8

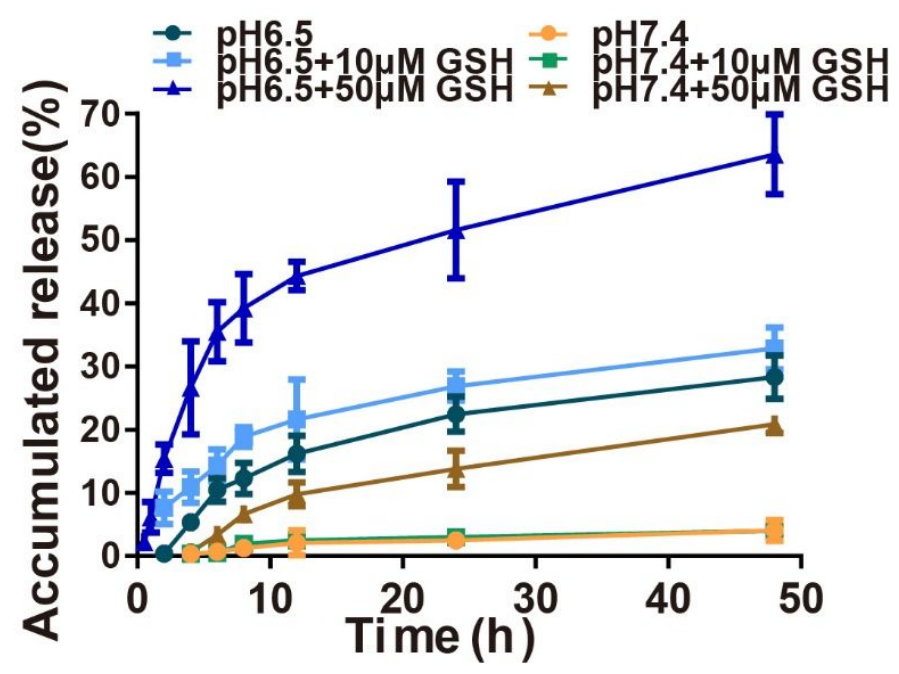

Figure S9

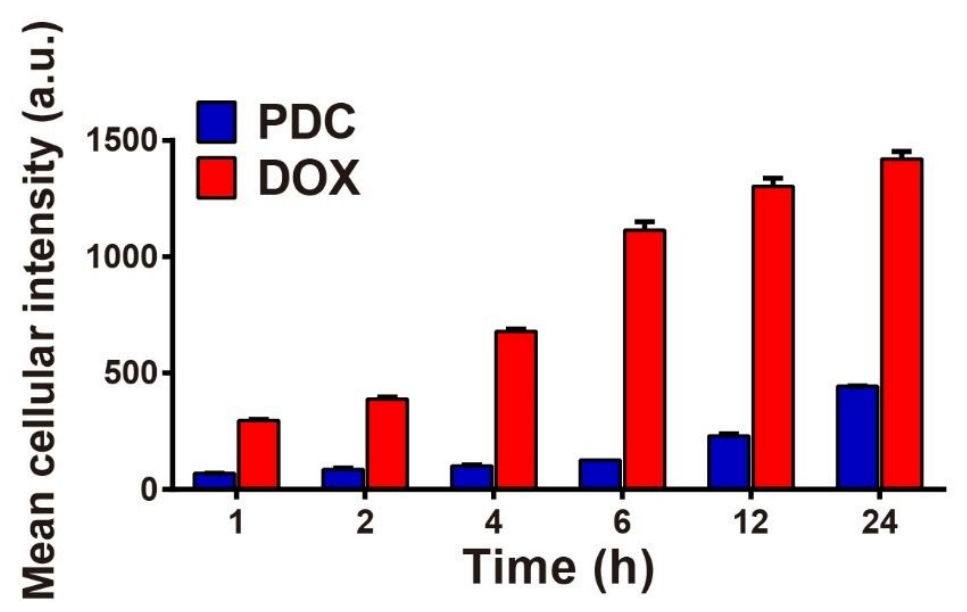


Figure S10

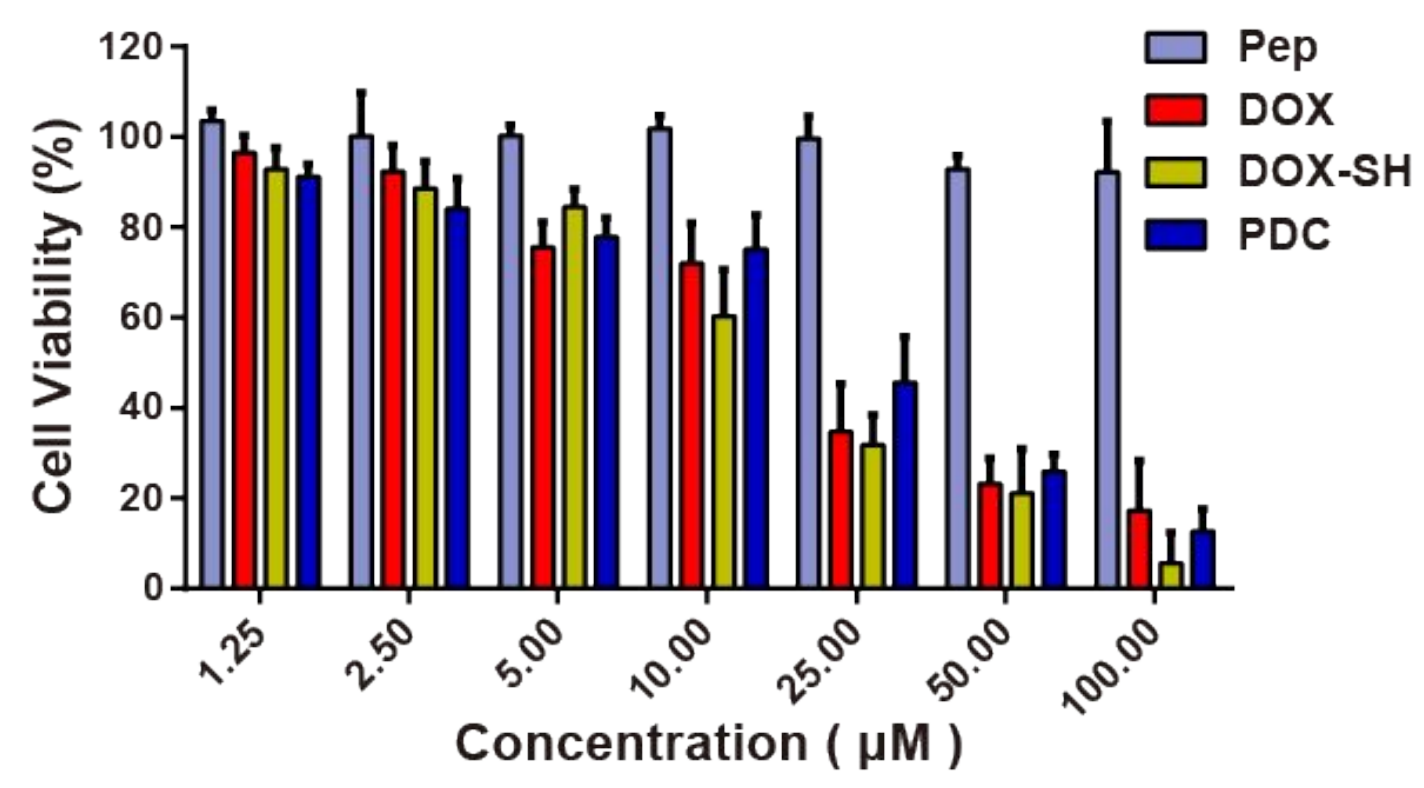

Figure S11

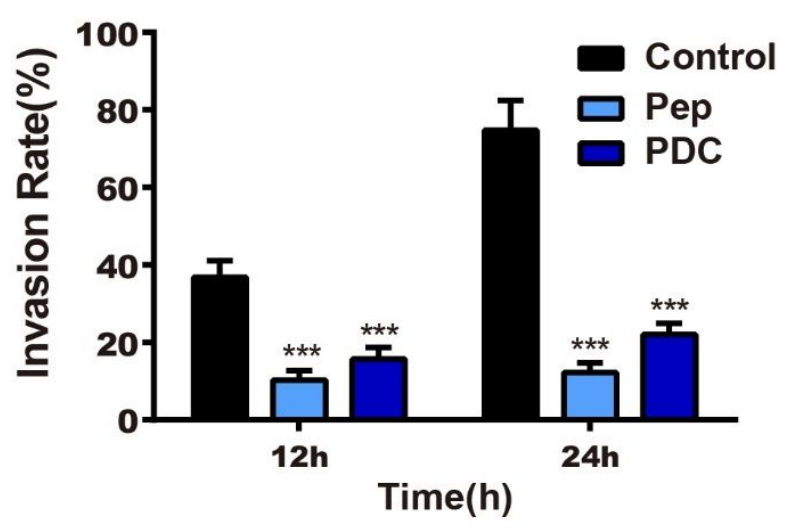

Figure S12

\section{Control}

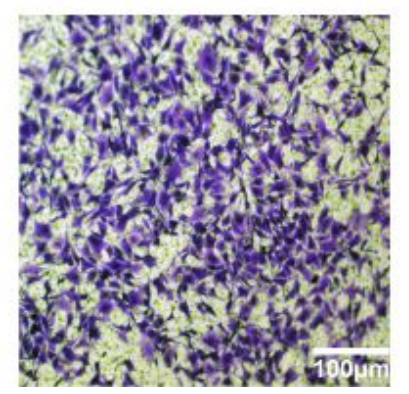

Batimastat

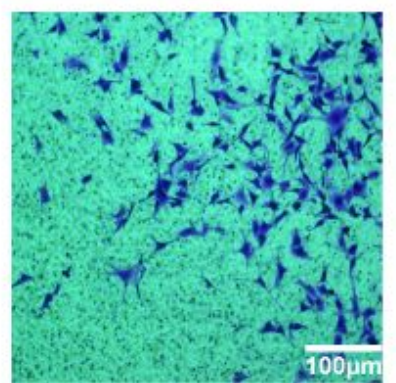

Figure S13 


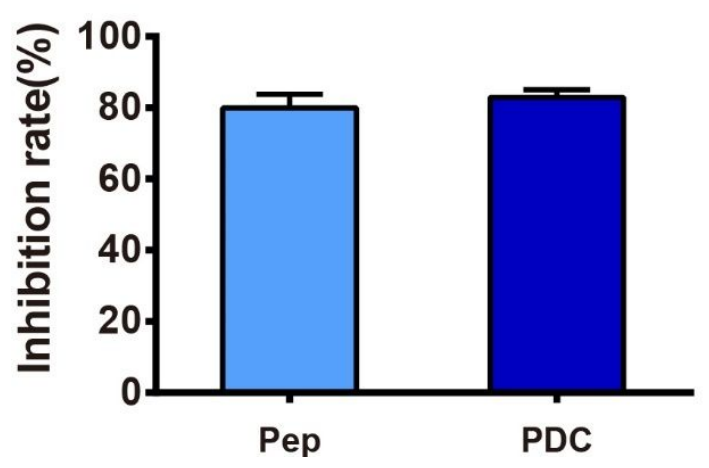

Figure S14

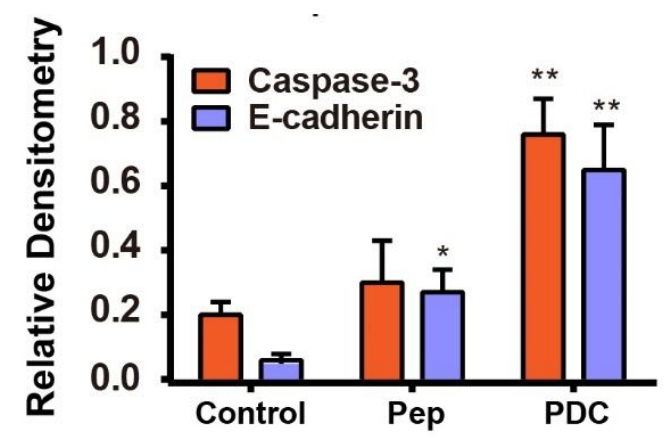

Figure S15

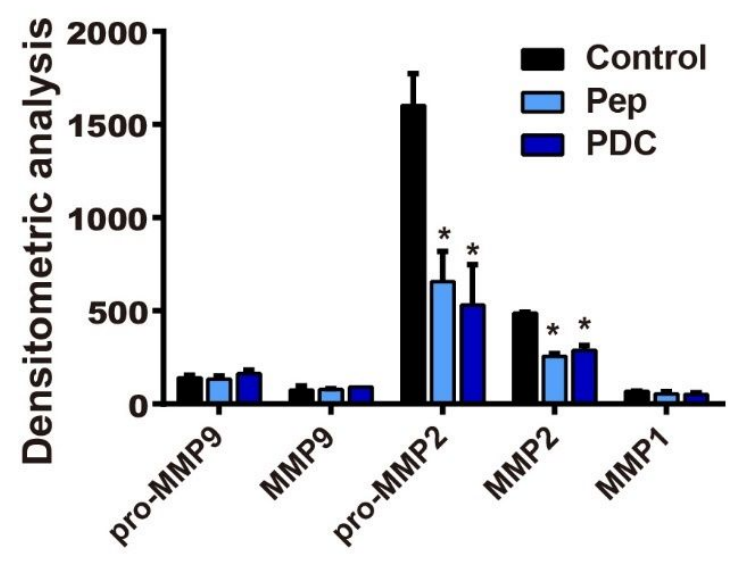

Figure S16 


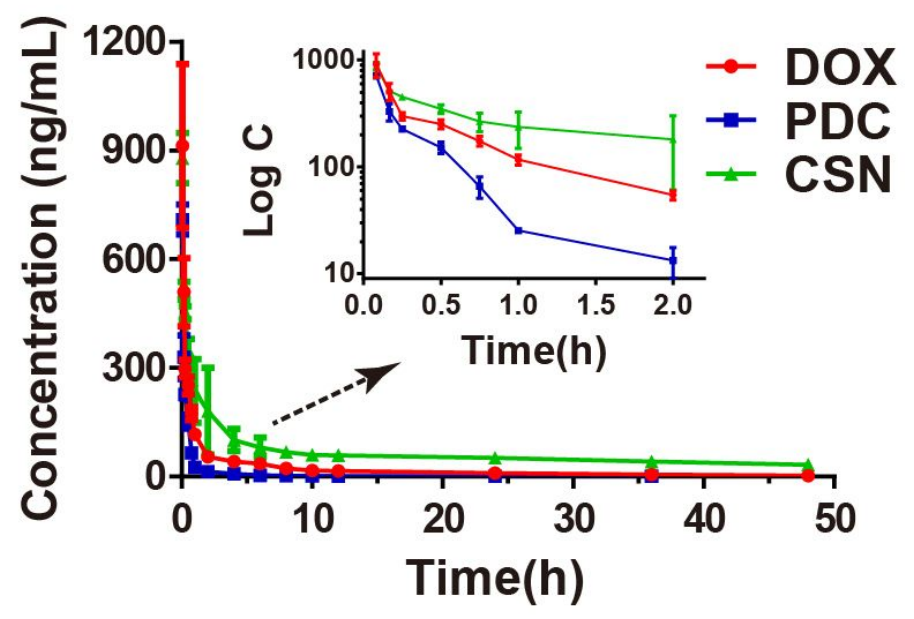

Figure S17
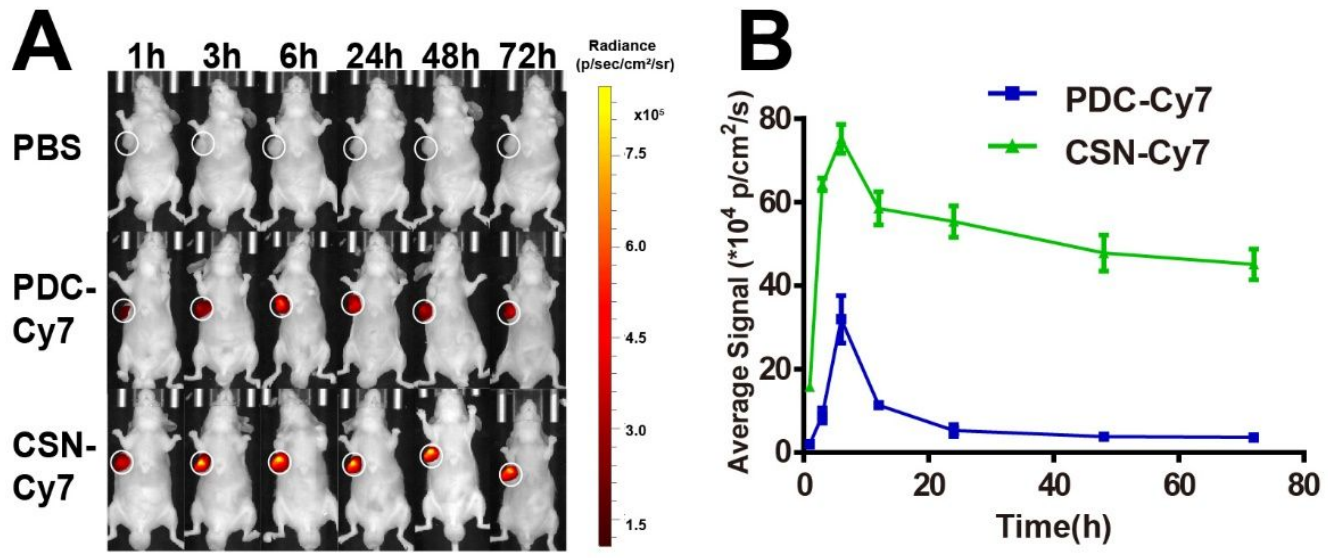

Figure S18

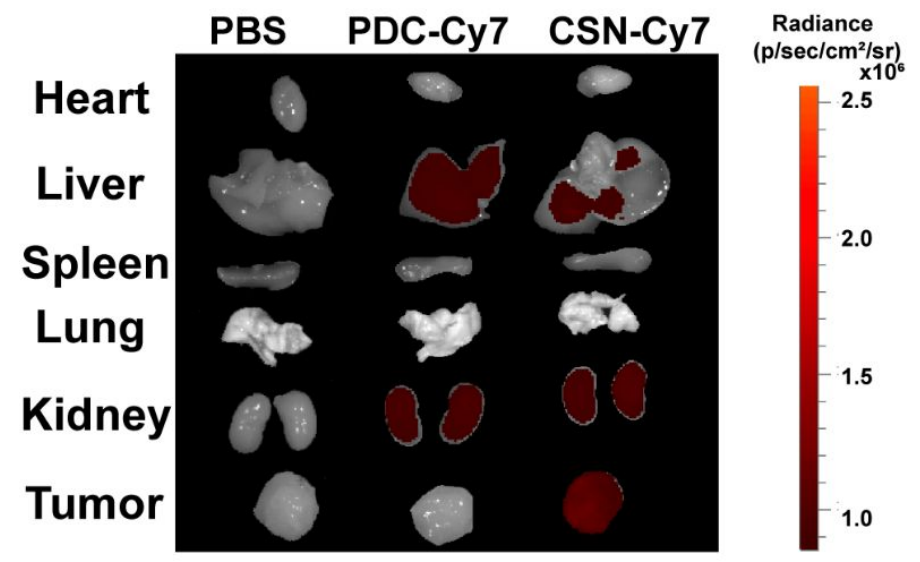

Figure S19 


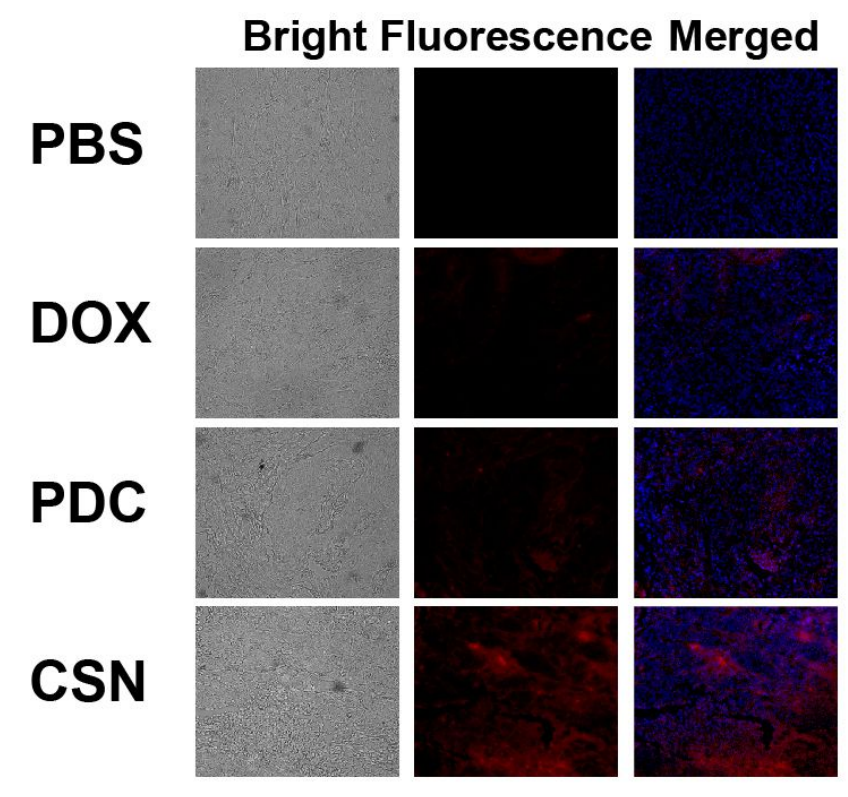

Figure S20

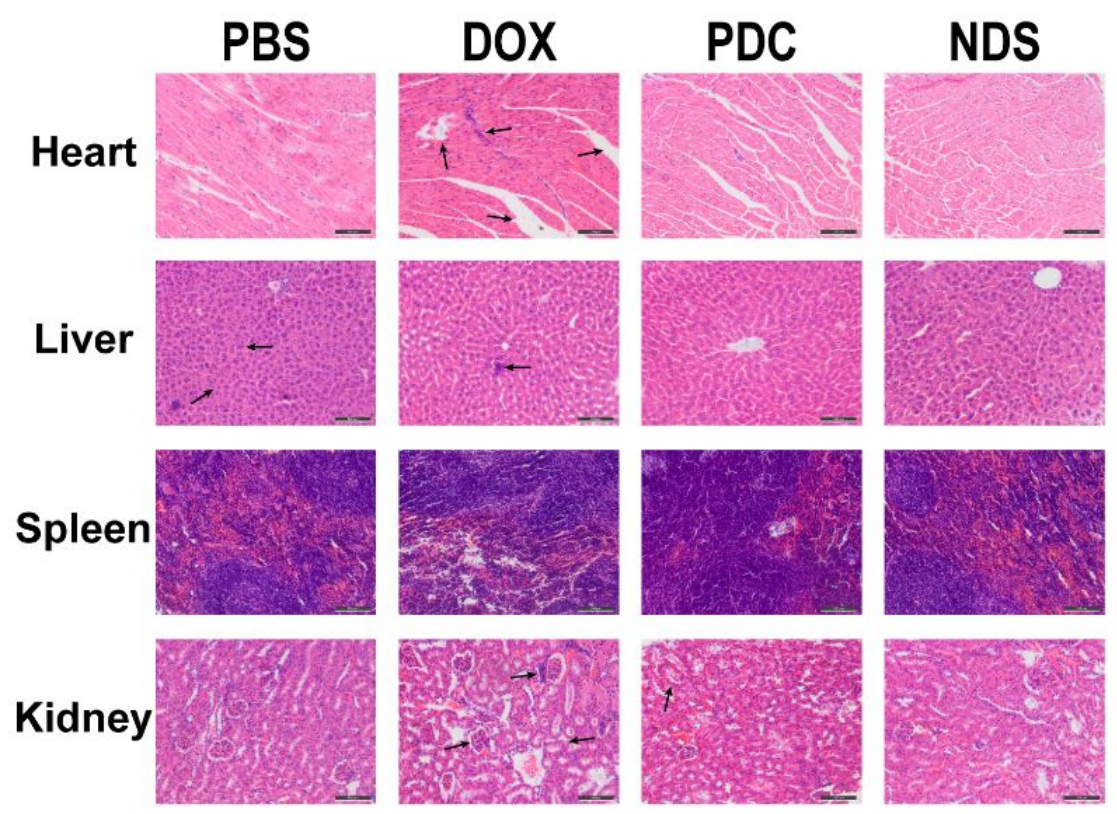




\section{Table S1}

\begin{tabular}{cccc}
\hline Parameters & DOX & PDC & CSNs \\
\hline $\mathrm{AUC}_{(0-\mathrm{t})}(\mathrm{ng} / \mathrm{mL} * \mathrm{~h})$ & $1033.59 \pm 115.64$ & $306.07 \pm 4.28$ & $3144.99 \pm 501.4$ \\
$\mathrm{AUC}_{(0-\infty)}(\mathrm{ng} / \mathrm{mL} * \mathrm{~h})$ & $1092.04 \pm 142.33$ & $307.43 \pm 3.88$ & $5369.39 \pm 571.65$ \\
$\mathrm{MRT}_{(0-\mathrm{t})}(\mathrm{h})$ & $8.65 \pm 0.26$ & $1.58 \pm 0.82$ & $16.63 \pm 2.30$ \\
$\mathrm{MRT}_{(0-\infty)}(\mathrm{h})$ & $11.73 \pm 1.03$ & $1.71 \pm 0.86$ & $59.50 \pm 29.26$ \\
$\mathrm{~T}_{1 / 2 \mathrm{z}}(\mathrm{h})$ & $13.30 \pm 2.52$ & $4.15 \pm 2.98$ & $47.64 \pm 20.59$ \\
$\mathrm{C}_{\text {max }}(\mathrm{ng} / \mathrm{mL})$ & $913.15 \pm 226.60$ & $709.70 \pm 41.58$ & $879.19 \pm 69.03$ \\
$\mathrm{CL}_{\mathrm{z}}(\mathrm{L} / \mathrm{h} / \mathrm{kg})$ & $4.63 \pm 0.58$ & $16.27 \pm 0.21$ & $0.94 \pm 0.10$ \\
$\mathrm{~V}_{\mathrm{z}}(\mathrm{L} / \mathrm{kg})$ & $87.41 \pm 5.04$ & $96.85 \pm 68.6$ & $62.90 \pm 20.98$ \\
\hline
\end{tabular}

Methods. The synthesis of DOX-SH. DOX and Traunt's reagents (2-iminothiocyclopentane hydrochloride) were dissolved in an appropriate methanol solvent at a molar ratio of 1:5; then, they were catalyzed by treatment with the appropriate amount of pyridine and they were allowed to react at $25^{\circ} \mathrm{C}$ for $12 \mathrm{~h}$. Most of the methanol was evaporated by rotary evaporation, which was followed by purification by preparative high-performance liquid chromatography. The target product, DOX-SH, was collected and freeze-dried. The purity (\%) of the DOX-SH was approximately 92.695 .

The synthesis of Pep. peptide C2KIGLFRWR and Py-S-S-Py (2,2' -dithiodipyridine) were dissolved in an appropriate amount of methanol solvent at a molar ratio of 1:3, and an appropriate amount of formic acid was added for catalysis. After reacting at $25^{\circ} \mathrm{C}$ for 2 days, the reaction solution was added dropwise to a large amount of ice ether under constant stirring, and then it was 
centrifuged for $5 \mathrm{~min}$. The precipitate was repeatedly cleaned with ice ether 3 times. The precipitate was dried in a vacuum drying oven, and Pep-S-S-Py was finally obtained. The purity (\%) of the active peptide was approximately 90.563 .

The synthesis of the PDC. DOX-SH and Pep-S-S-Py were dissolved in an appropriate amount of methanol solvent at a molar ratio of $2.5: 1$; catalysis was enabled by the addition of an appropriate amount of formic acid, and they were reacted at $25^{\circ} \mathrm{C}$ for 2 days. After the reaction, most of the methanol was evaporated by a rotatory steam, and the products were purified by preparative high performance liquid chromatography. The target products were collected and freeze-dried to obtain the PDC. (Figure S2). The purity (\%) of the PDC was approximately 93.513.

The synthesis of MPL. We modified polylysine (E-PL) with 2,3-dimethyl maleic anhydride (DMMA) and the amino group on the lysine side chain transformed to a amide bone and a negatively charged carboxyl group. A total of $0.165 \mathrm{mmol}$ of $\varepsilon$-PL was dissolved in $25 \mathrm{~mL}$ of deionized water, then $5 \mathrm{mmol}$ of DMMA, $5 \mathrm{mmol}$ of NHS, and $6 \mathrm{mmol}$ of EDCI were added to the $\varepsilon$-PL solution. Triethylamine was added to adjust the $\mathrm{pH}$ of the solution to 8.5 , and the solution was stirred at $25^{\circ} \mathrm{C}$ for $48 \mathrm{~h}$. Then, it was ultrafiltered with a dialysis tube (MW $3000 \mathrm{Da}$ ). After freeze-drying, $1200 \mathrm{mg}$ of white solid MPL was obtained.

Construction of CSNs. A PDC solution was dropwise added to MPL (Figure S3), and the final concentration of the PDC was $200 \mu \mathrm{M}$; the molar ratio of PDC: MPL $=1: 1.25$. Then, the mixture was stirred overnight.

Cellular uptake. SMMC-7721 cells were inoculated in 24 -well plates $\left(2 \times 10^{5}\right.$ cells/well). The DOX, PDC, and PDC with GSH were added to the cells at a final DOX concentration of 10 $\mu \mathrm{M}$. After incubating the cells and drugs for different amounts of time, the medicated cells were 
washed with sterile PBS many times to remove any drugs on the surface of the plate or cell membrane. After trypsin digestion, the cells were centrifuged, washed, counted and finally suspended in $1 \mathrm{~mL}$ of PBS with $10^{6}$ cells. The average fluorescence intensity of $10^{4}$ cells in a gated region was used to estimate intracellular intake, as measured by a flow cytometer (Guava easyCyte).

MTT colorimetric assay. SMMC-7721 cells were added in $96-$ well plate $\left(1 \times 10^{4}\right.$ cells/well). The incubation condition is $37^{\circ} \mathrm{C}$ and $5 \% \mathrm{CO}_{2}$ with saturated humidity. After $24 \mathrm{~h}$, different concentrations of Pep, DOX, DOX-SH, and PDC were added to the 96-well plate. After $24 \mathrm{~h}$ of culture, the supernatant was discarded. Add $100 \mu \mathrm{L}$ of MTT solution to the well, and they were incubated together for $4 \mathrm{~h}$. Add $150 \mu \mathrm{L}$ of DMSO to dissolve the product, and the absorbance was measured at $450 \mathrm{~nm}$ to calculate the cell survival rate.

Cell migration assay. Mark with a marker pen on the six-well plate to determine the cell location. SMCC7721 cells in good growth state are seeded at $4 \times 10^{5}$ per well and serum-free cultured for $12 \mathrm{~h}$ to reduce the effect of cell proliferation. Evenly scratch the layer of cells with the tip of the micropipette. Then the cells were cultured with batimastat $(1 \mu \mathrm{M})$, Pep $(100 \mu \mathrm{M})$, and CSNs (10 $\mu \mathrm{M})$. After incubation for $0,12,24 \mathrm{~h}$, the degree of cell healing was observed by an inverted microscope at 40 times magnification. The changes in migration were measured by ImageJ software.

Transwell matrix invasion assay. A layer of matrigel is laid on the upper compartment side of the polycarbonate film to simulate the extracellular matrix in vivo. Before cells enter the lower compartment, they first secreted MMPs to degrade the matrigel and then pass through the polycarbonate film. Add $100 \mathrm{uL}$ of Matrigel gel diluted with serum-free cold cell culture medium to the 24-well transwell chamber and incubate at $37{ }^{\circ} \mathrm{C}$ for at least $30 \mathrm{~min}$. The gel was gently washed with serum-free medium to hydrate the basement membrane. SMMC-7721 cells were 
seeded in the upper chamber $\left(1 \times 10^{4}\right.$ cells/well $)$. Batimastat $(1 \mu \mathrm{M})$, Pep $(10 \mu \mathrm{M})$, and PDC $(10 \mu \mathrm{M})$ were dissolved in the upper chamber. After $24 \mathrm{~h}$, remove the Transwell chamber, blot the liquid from the upper chamber, wash it twice with calcium-free PBS, transfer to a well with approximately $800 \mu \mathrm{L}$ of methanol added in advance, and fix at $25^{\circ} \mathrm{C}$ for $30 \mathrm{~min}$. Put into $800 \mu \mathrm{L}$ of $0.1 \%$ crystal violet staining solution in wells, and incubate for $30 \mathrm{~min}$. Use a cotton swab to gently wipe off the upper non-migrating cells and wash them with PBS 3 times. Four fields were observed on a $400 \times$ microscope. The dye was fully dissolved with $33 \%$ acetic acid, and the OD value of the marker was measured at $570 \mathrm{~nm}$ to indirectly reflect the number of cells.

Western blot analysis. Use RIPA buffer solution to lyse the cells after drug incubation, The BCA protein kit was used for quantitative protein detection. According to the molecular weight of the E-cadherin and caspase $3,10 \%$ and $12 \%$ SDS-PAGE were selected for protein separation. After blocking, the membrane was washed with TBST. The membrane was placed in a plate containing a primary antibody and incubated. Next day, wash the membrane three times, add diluted secondary antibody, and shake at $25^{\circ} \mathrm{C}$ for $2 \mathrm{~h}$. The target protein was stained using a chemiluminescence kit, and a blot image was obtained using an imaging system (SYNGENE, G:BOXChemiXR5, UK). The values of the bands were calculated using gel optical density analysis software Gel-Pro 4.0.

Gelatin zymography. The cell supernatant was extracted and separated by electrophoresis for $1.5 \mathrm{~h}$ with $10 \%$ SDS-PAGE containing $0.8 \%$ gelatin and $0.2 \%$ collagen. After electrophoresis, the gel was eluted twice with $2.5 \%$ Triton X-100 for $40 \mathrm{~min}$. After incubation for $42 \mathrm{~h}$, use Coomassie blue to dye for $3 \mathrm{~h}$. Finally, the decolorization solution with methanol concentration of $30 \%, 20 \%, 10 \%$, and acetic acid concentration of $15 \%, 10 \%$, and $5 \%$ was used for $2 \mathrm{~h}$, 
respectively. The MMPs activity was quantitatively analyzed by Gel-Pro 4.0 software.

Pharmacokinetics. The experimental protocols were approved by the Animal Care and Use Committee of Nanjing University of Chinese Medicine. The 18 male SD rats were divided into three groups randomly $(\mathrm{n}=6)$, and DOX, PDC, and CSNs solution (dose equivalent of $4 \mathrm{mg} / \mathrm{kg}$ DOX) were injected into tail vein, respectively. 15 drops of orbital blood were taken and placed in a $1.5 \mathrm{~mL}$ centrifuge tube soaked with heparin sodium at $0,0.083,0.17,0.25,0.5,0.75,1,2,4,6,8$, 10, 12, 24, 36, and $48 \mathrm{~h}$ after dosing. The upper plasma was centrifuged (4000 rpm, $10 \mathrm{~min}$ ). Add $90 \mu \mathrm{L}$ of plasma sample, $10 \mu \mathrm{L}$ of clarithromycin internal standard solution to the thickened centrifuge tube, add $300 \mu \mathrm{L}$ of acetonitrile to precipitate the protein, and vortex for $10 \mathrm{~min}$. Centrifuge at $13000 \mathrm{r} / \mathrm{min}$ for $5 \mathrm{~min}$, carefully suck the supernatant into another clean centrifuge tube, and repeat the centrifugation three times according to the above requirements, and take 180 $\mu \mathrm{L}$ of the supernatant for analysis by UHPLC-MS/MS. The linear ranges of DOX and PDC were $\mathrm{y}=0.03617 \mathrm{x}+0.23715(5-2500 \mathrm{ng} / \mathrm{mL})$ and $\mathrm{y}=0.00135 \mathrm{x}+0.03912(10-1000 \mathrm{ng} / \mathrm{mL})$, respectively. Pharmacokinetic parameters, including AUC, MRT, $\mathrm{t}_{1 / 2}, \mathrm{C}_{\max }, \mathrm{CL}$, and $\mathrm{V}$, were analyzed by DAS2.0 software.

Animal Model. The HCCLM3 cell pellet was collected. Matrigel was diluted in ice-cold PBS at a ratio of 1:1. The cell density was adjusted with diluted Matrigel for inoculation of BALB/c male nude mice at $5 \times 10^{6}$ cells $/ 150 \mu \mathrm{L}$. Twenty nude mice were inoculated in the right side of the armpit. Eighteen nude mice that formed tumors were divided into 3 groups randomly after 10 days: the PBS group, DOX group, and CSNs group. The mice were administered to the caudal vein every 3 days for 24 days. The dose used for DOX was $6 \mathrm{mg} / \mathrm{kg}$. Tumor size and body weight were assessed every 2 days. Every 6 days, mice were injected with D-luciferin intraperitoneally at a concentration 
of $10 \mu \mathrm{L} / \mathrm{g}$, and $15 \mathrm{~min}$ after the injection, the tumor growth, and metastasis were photographed by biofluorescence. The mice were sacrificed on day 27 , and the tumor size (width and length) was measured. $\mathrm{V}\left(\mathrm{mm}^{3}\right)=\operatorname{width}^{2}\left(\mathrm{~mm}^{2}\right) \times$ length $(\mathrm{mm}) / 2$.

Statistical Analysis. SPSS software (version 16.0, USA) was used for analysis. Data are given as mean $\pm \mathrm{SD}$. Statistical significance was analyzed by one-way ANOVA or Student's t-tests and accepted at $\mathrm{p}<0.05\left(* P<0.05,{ }^{* *} P<0.01\right.$, and $\left.* * * P<0.001\right)$. 